\title{
The Impact of Heat-Moisture Treatment on the Properties of Musa paradisiaca L. Starch and Optimization of Process Variables
}

\section{Maria José Missão \\ Cordeiro', Cristiane Martins \\ Veloso ${ }^{1 *}$, Leandro Soares \\ Santos ${ }^{2}$, Renata Cristina \\ Ferreira Bonomo', Márcio \\ Caliari and Rafael da \\ Costa Ilhéu Fontan}

'Process Engineering Laboratory, State University of Southwest Bahia, 45700-000 Itapetinga, BA, Brazil

${ }^{2}$ Agronomy School, Federal University of Goiás, Campus Samambaia, 74690900 Goiânia, GO, Brazil

${ }^{*}$ Corresponding author:

Phone: +557732618659;

E-mail: crismveloso@yahoo.com.br

ORCID IDs: 0000-0002-7041-9451 (Cordeiro), 0000-0001-5702-3500 (Veloso), 0000-0001-7431-318X (Santos), 0000-0002-9896-4099 (Bonomo), 0000-0002-0877-8250 (Caliari), 0000-0001-9982-3418 (Fontan)

\section{SUMMARY}

Starch stability under the processing conditions can be improved by modifying the granule structure using chemical and/or physical processes. The effect of heat-moisture treatment (HMT) on the physicochemical, morphological, pasting and thermal properties of green banana (Musa paradisiaca L.) starch was investigated. To analyze the changes in starch properties due to the combined effect of the process variables, time $(\mathrm{h})$, moisture $(\%)$, and temperature $\left({ }^{\circ} \mathrm{C}\right)$ were considered as independent variables using a central composite rotatable design. The native starch extracted using ammonium hydroxide as an antioxidant contained $80.4 \%$ total carbohydrates, $53.7 \%$ apparent amylose, $11.46 \%$ moisture, and other constituents (ash, protein, lipids), which accounted for less than $1 \%$. The granule morphology was affected by the moisture and temperature used in HMT. A and $B$ type X-ray diffraction patterns were observed in the native and modified starch. Mathematical models that describe the behaviour of modified starch properties as a function of the evaluated parameters were obtained. The variables time and temperature significantly affected the physicochemical, rheological and digestibility properties of starch.

Key words: Musa paradisiaca L., physical changes, physicochemical properties, thermal properties, digestibility

\section{INTRODUCTION}

The main sources of commercial starch are corn, wheat, potato and cassava. However, there is a trend towards different sources of natural starch with characteristics and properties that meet the consumer demand and different industrial segments. Thus, the processing of unexplored raw materials, like the starch extracted from green banana (Musa paradisiaca $\mathrm{L}$.), has been studied to assess its properties for industrial applications. The green banana starch exhibits physicochemical, functional and digestibility characteristics different from those of conventional sources such as corn, wheat, rice and potato (1). This starch has high concentration of indigestible or resistant starch fraction, which presents health benefits and physiological improvements, controlling or preventing diseases, with important applications in processed foods, making them more nutritious and commercially viable. In addition, bananas have low production costs, and the starch extraction may be a viable alternative to minimize post-harvest losses (2).

Several factors can impair the industrial applications of native starch, including low shear stress resistance, thermal decomposition, high retrogradation and syneresis, and structure instability under different temperatures, $\mathrm{pH}$, and pressure conditions (3). However, the polysaccharide molecule can be modified to reach the desirable characteristics, for example using a physical method, such as heat-moisture treatment (HMT), once no chemical reagents are used and the resulting product does not need to be classified as a modified starch for commercialization (4).

The HMT involves the treatment of starch under insufficient moisture and temperature values higher than those of gelatinization for a given period of time, leading to structural modifications in the granules without destruction of the structure, with significant impact on the general properties, including starch digestibility (3-5). 
Several authors have studied heat treatment to modify starch granules, including rice starch (6), potato starch (5), cashew nut starch (7), Morato banana starch (8), and corn starch (9). However, statistical tools have not been used to optimize variables that influence the modification process (i.e. HTM). Although most studies have evaluated the specific and non-systematic conditions of hydrothermal treatment (temperature, time and moisture content), changes in all the operating conditions have not been assessed. In this context, this study evaluates the effect of temperature, time and moisture on physicochemical, morphological, pasting and thermal properties of banana (Musa paradisiaca L.) starch using the central composite rotatable design (CCRD) as optimization method.

\section{MATERIALS AND METHODS}

\section{Starch extraction}

Green banana (Musa paradisiaca L.) was purchased from the local grower (Bahia, Brazil), and standardized according to the maturity degree, with a completely green peel. Starch was extracted as reported by Moorthy (10), with some modifications, using $0.03 \mathrm{~mol} / \mathrm{L}$ ammonium hydroxide as an antioxidant at a ratio of 1:7 $(\mathrm{m} / \mathrm{V})$. The green banana was triturated in a domestic blender (Philips Walita, Varginha, MG, Brazil), followed by successive washing with distilled water and centrifugation at 3000×g (Q222tm2; Quimis, Diadema, SP, Brazil) until the supernatant was clear. Then, the resulting mass was dried in a forced air circulation oven TE-394/1 (Tecnal, São Paulo, SP, Brazil) at $40^{\circ} \mathrm{C}$ for $24 \mathrm{~h}$.

\section{Heat-moisture treatment}

The heat-moisture treatment (HMT) of banana (Musa paradisiaca L.) starch was performed according to the method described by Chung et al. (11). Starch was subjected to different moisture levels, temperature, and time conditions. For that, the initial moisture content of the native starch was determined, which was subsequently adjusted by the addition of distilled water to reach the desired level, according to the central composite rotatable design (CCRD) (Table 1), using the following equation:

$$
m_{\mathrm{w}}=\left[\left(\frac{100-\mathrm{M}_{\mathrm{i}}}{100-\mathrm{M}_{\mathrm{f}}}\right)-1\right] \cdot \mathrm{m}
$$

where $m_{\mathrm{w}}(\mathrm{g})$ is the mass of water to be added, $M_{\mathrm{i}}(\%)$ is the initial moisture of the sample, $M_{\mathrm{f}}(\%)$ is the desired moisture, and $m(\mathrm{~g})$ is the sample mass.

A mass of $60 \mathrm{~g}$ native starch was placed in a stainless steel reactor, and distilled water, according to the experimental planning shown in Table 1, was added and mixed. The sample remained in the reactor at $4{ }^{\circ} \mathrm{C}$ for $24 \mathrm{~h}$. The hermetically sealed samples were oven-heated at the set temperatures and times. Starch samples were dried in an oven TE-394/1 (Tecnal) with forced air circulation at $40{ }^{\circ} \mathrm{C}$ until reaching a moisture content close to $12 \%$.

\section{Physicochemical characterization}

The moisture content (AOAC method 945.15) (12), ash (AOAC method 923.03) (13), protein (AOAC method 945.18) (14) and $\mathrm{pH}$ (AOAC method 945.27) (15) values were determined according to AOAC.

The total lipids were determined as described by Bligh and Dyer (16) using $5 \mathrm{~g}$ of starch and a homogeneous solution of chloroform, methanol and water in a ratio 1:2:0.8, respectively. The apparent amylose content was measured by the colorimetric method, which is based on the transmission of light through the coloured complex the amylose forms when reacting with iodine, according to the method of Martinez et al. (17). The water absorption index was determined at $30^{\circ} \mathrm{C}$ by the method proposed by Kaitaranta et al. (18), while swelling power and solubility were determined at $95^{\circ} \mathrm{C}$ according to the method of Schoch (19).

Resistant starch and non-resistant starch were determined using the Megazyme International Reagent Kit (Bray, Ireland), according to the method described by the manufacturer. Samples were incubated in a shaking water bath (Q215m2; Quimis) at $200 \mathrm{rpm}$ with pancreatic a-amylase and amyloglucosidase at $37^{\circ} \mathrm{C}$ for $16 \mathrm{~h}$, during which time non-resistant starch was solubilised and hydrolysed to D-glucose by the combined action of the two enzymes. The reaction was terminated by the addition of an equal volume of ethanol or industrial methylated spirits (IMS, denatured ethanol), and the resistant starch was recovered as pellets after centrifugation at $1000 \times g$ (Q222tm2; Quimis). The pellets were then washed twice with the suspension of aqueous IMS or ethanol (50\%, by volume), and separated by centrifugation at $3000 \times g$. Free liquid was removed by decantation. Resistant starch pellets were dissolved in $2 \mathrm{~mol} / \mathrm{L} \mathrm{KOH} \mathrm{P.A.-A.C.S}$ (Synth, Diadema, SP, Brazil), by vigorously stirring at 1000 $\times g$ in an ice-water bath over a magnetic stirrer (Q215m2; Quimis). The solution was then neutralised with acetate buffer (Synth) and the starch was quantitatively hydrolysed to glucose with amyloglucosidase. D-Glucose was measured with glucose oxidase/peroxidase reagent (GOPOD; Megazyme), and this was a measure of the resistant starch content of the sample. Non-resistant starch (solubilised starch) was determined by pooling the original supernatant and washings with distillated water, adjusting the volume to $100 \mathrm{~mL}$ and measuring D-glucose content with GOPOD.

\section{$X$-ray diffraction analysis}

X-ray diffraction (XRD) analysis of starch was performed in a Bruker D2 Phaser diffractometer (Karlsruhe, Germany) using CuKa ( $30 \mathrm{kV}$ and $10 \mathrm{~mA}$ ) radiation in the range of $5^{\circ} \leq 2 \theta \leq 40^{\circ}$, at $1600 \mathrm{~V}$, in a continuous step.

\section{Pasting properties of green banana starch}

The pasting properties of green banana (Musa paradisiaca L.) starch were determined in a rapid visco analyzer (RVA 4500; Perten Instruments, PerkinElmer, Hägersten, Sweden), according to Hart Weber et al. (20), with modifications. Starch suspensions at the concentration of $0.1 \mathrm{~g} / \mathrm{mL}$ were analysed with RVA 
Table 1. Central composite rotatable design (CCRD) of the experiment

\begin{tabular}{|c|c|c|c|c|c|c|c|}
\hline & $\mathrm{X} 1$ & $X 2$ & X3 & & & \\
\hline & & Temperature & $t$ & $w$ (moisture) & & & \\
\hline & & ${ }^{\circ} \mathrm{C}$ & $\mathrm{h}$ & $\%$ & & & \\
\hline \multicolumn{2}{|r|}{$\mathrm{CP}$} & 100 & 13 & 25 & & & \\
\hline \multicolumn{2}{|r|}{ Delta } & 12 & 6.5 & 6 & & & \\
\hline \multirow{3}{*}{ Trial } & \multirow{3}{*}{ Repetition } & \multicolumn{3}{|c|}{ Coded level } & \multicolumn{3}{|c|}{ Actual level } \\
\hline & & $x_{1}$ & $x_{2}$ & $x 3$ & Temperature & $t$ & $w($ moisture $)$ \\
\hline & & X1 & $x 2$ & X3 & ${ }^{\circ} \mathrm{C}$ & $\mathrm{h}$ & $\%$ \\
\hline 1 & 1 & -1 & -1 & -1 & 88 & 6.5 & 19 \\
\hline 2 & 1 & 1 & -1 & -1 & 112 & 6.5 & 19 \\
\hline 3 & 1 & -1 & 1 & -1 & 88 & 19.5 & 19 \\
\hline 4 & 1 & 1 & 1 & -1 & 112 & 19.5 & 19 \\
\hline 5 & 1 & -1 & -1 & 1 & 88 & 6.5 & 31 \\
\hline 6 & 1 & 1 & -1 & 1 & 112 & 6.5 & 31 \\
\hline 7 & 1 & -1 & 1 & 1 & 88 & 19.5 & 31 \\
\hline 8 & 1 & 1 & 1 & 1 & 112 & 19.5 & 31 \\
\hline 9 & 1 & -1.68 & 0 & 0 & 79.84 & 13 & 25 \\
\hline 10 & 1 & 1.68 & 0 & 0 & 120.16 & 13 & 25 \\
\hline 11 & 1 & 0 & -1.68 & 0 & 100 & 2.08 & 25 \\
\hline 12 & 1 & 0 & 1.68 & 0 & 100 & 23.92 & 25 \\
\hline 13 & 1 & 0 & 0 & -1.68 & 100 & 13 & 14.92 \\
\hline 14 & 1 & 0 & 0 & 1.68 & 100 & 13 & 35.08 \\
\hline 15 & 1 & 0 & 0 & 0 & 100 & 13 & 25 \\
\hline 15 & 2 & 0 & 0 & 0 & 100 & 13 & 25 \\
\hline 15 & 3 & 0 & 0 & 0 & 100 & 13 & 25 \\
\hline 15 & 4 & 0 & 0 & 0 & 100 & 13 & 25 \\
\hline 15 & 5 & 0 & 0 & 0 & 100 & 13 & 25 \\
\hline
\end{tabular}

calibrated at $50{ }^{\circ} \mathrm{C}$ for $1 \mathrm{~min}$, followed by heating to $95^{\circ} \mathrm{C}$ at a rate of $6^{\circ} \mathrm{C} / \mathrm{min}$ for $5 \mathrm{~min}$, and cooling to $50^{\circ} \mathrm{C}$ at a rate of $6^{\circ} \mathrm{C} /$ $\min$. The measured parameters were: pasting temperature $\left({ }^{\circ} \mathrm{C}\right)$, maximum viscosity (mPa.s), final viscosity $(\mathrm{mPa} \cdot \mathrm{s})$, breakdown temperature $\left({ }^{\circ} \mathrm{C}\right)$, and setback viscosity (mPa.s).

\section{Starch thermal properties}

Starch gelatinization was evaluated using a differential scanning calorimeter (DSC model Q20; TA Instruments, New Castle, DE, USA), as described by Hart Weber et al. (20), with modifications. For that, $2.0 \mathrm{mg}$ (on dry basis) sample was weighed into aluminum pans and $6 \mu \mathrm{L}$ distilled water were added. The pans were hermetically sealed and held for $12 \mathrm{~h}$ at room temperature, and then brought to the equipment where they were subjected to a heating cycle from 40 to 120 ${ }^{\circ} \mathrm{C}$ at a rate of $10^{\circ} \mathrm{C} / \mathrm{min}$. An empty hermetically sealed aluminum pan was used as a reference, the DSC cell was calibrated with indium, and the experiment was performed under a dynamic nitrogen atmosphere $(50 \mathrm{~mL} / \mathrm{min})$.

\section{Morphological properties}

The morphological properties of granules were evaluated by scanning electron (SEM, JSM-6610; JEOL Ltd., Tokyo, Japan) equipped with energy-dispersive spectroscopy (EDS, EDS NSS
2.3; Thermo Fisher Scientific, Waltham, MA, USA), operating at $5 \mathrm{kV}$, with acquisition software Scanning Electron Microscope (SEM) Control User Interface v. 2.24 (21). The samples were mounted on the stubs using double-sided conductive tape, the excess was sprayed with $\mathrm{CO}_{2}$ and then placed in Desk V apparatus (Denton Vacuum, LLC, Moorestown, NJ, USA) for gold deposition for $2 \mathrm{~min}$, using gold as the conductive metal. These analyses were performed in the High Resolution Microscopy Multiuser Laboratory (LabMic), at the Institute of Physics, UFG (Federal University of Goiás, Goiânia, Brazil).

\section{Statistical analysis}

To analyze the effect of the heat treatment on the starch properties, a central composite rotatable design (CCRD) was used with 19 trials and 15 treatments, and five replicates at the central point (Table 1). The results were subjected to regression analysis to evaluate the effect of treatments and variables, with their respective interactions. The regression coefficients were estimated from the experimental data by multiple linear regression and a second-order polynomial model. The regression coefficients of quadratic model were tested, and the model with significance ( $\mathrm{p} \leq 0.05)$, non-significant lack of adjustment ( $p>0.05)$ and suitability of the model to the phenomenon was selected. All analyses were performed using the SAS software, version University Edition (22). 


\section{RESULTS AND DISCUSSION}

\section{Characterization of native starch}

The extraction yield (19.4\%) indicated the industrial potential of green banana as a starch source when compared to the varieties commonly used, such as sweet potato (18\%), arrowroots (8-16\%), yam (18-23\%), and Peruvian carrot (5-23 $\%)$ (23). Native starch had a moisture content of $11.48 \mathrm{~g} / 100$ $\mathrm{g}$, and low protein $(0.06 \mathrm{~g} / 100 \mathrm{~g})$, lipid $(<0.1 \mathrm{~g} / 100 \mathrm{~g})$, and ash $(0.06 \mathrm{~g} / 100 \mathrm{~g})$ levels, and total carbohydrate content of 88.4 $\mathrm{g} / 100 \mathrm{~g}$. The $\mathrm{pH}$ remained alkaline (8.26), between 9 and 10, similar to the $\mathrm{pH}$ of the pulp, as reported in the literature (10). The starch had a high apparent amylose content on dry mass bases $(53.7 \mathrm{~g} / 100 \mathrm{~g})$, probably due to the used method, which measures a blue complex formed by the classical reaction between the $a-1,4$ bonds of amylose and iodine. The extent of this complex may vary essentially based on the characteristics of its formation, depending on the molecular mass of the amylose and the concentration of iodide, increasing the absorbance with the increase in these variables (24).

\section{Morphology of native and heat-and-moisture-treated green banana starch granules}

The effect of the variables time, temperature and moisture during the heat treatment on granule morphology was observed by scanning electron microscopy (Fig. 1). The granules had some imperfections on the surface (Fig. 1c) when compared to the native starch (Fig. 1a), which had a smooth and uniform surface. This can be explained by the high temperature and moisture content used during the modification, which possibly caused pre-gelatinization of starch, evidenced

a)

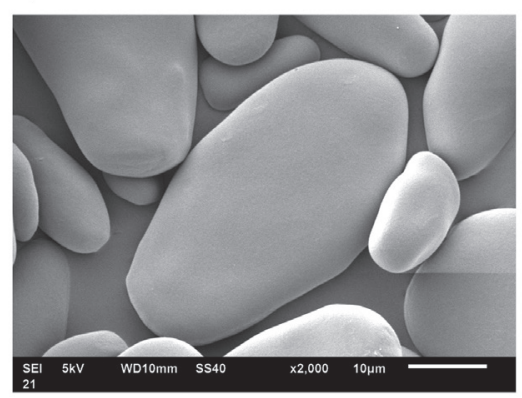

c)

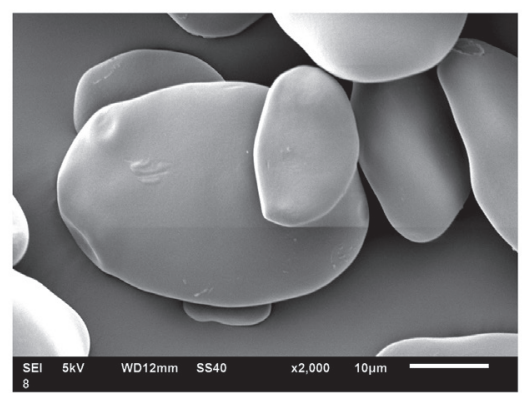

by the apparent deformity in the outer structure of the granules, being less pronounced in the samples of trials 1 (Fig. 1b) and 12 (Fig. 1d), which were subjected to milder temperature and moisture conditions. The observed physical changes were possibly a result of the partial melting and restructuring of the amylose and amylopectin chains on the granule surface (4).

\section{Physicochemical characterization of heat-and- -moisture-treated starch}

In the diffractogram of the native starch, well-defined peaks (2 $\theta$ ) were observed at $11^{\circ}, 15^{\circ}, 17^{\circ}, 20^{\circ}, 23^{\circ}$ and $26^{\circ}$, characterizing starch types A and B (25). After modification, all starch exhibited diffraction peaks at $17^{\circ}$ and $18^{\circ}$, an unfolding of native starch peak at $17^{\circ}$, with an A-type crystalline structure typical of gelatinized starch $(26,27)$. This characteristic is in accordance with the modifications observed in the morphology of HMT starch, which was compatible with the pregelatinized starch. The modified starch showed higher crystallinity, as demonstrated by the peak shape at $23^{\circ}$. Changes in crystallinity after HMT depends on the botanical origin and the conditions used in the treatment (24). The increase in crystallinity of the starch after the applied treatments may be related to the high concentration of apparent amylose, and in the native starch it is mainly associated with the amorphous region, which has a vitreous nature. That may result in a granular structure of the native starch. However, after gelatinization, the amylose plays a role in the crystallization due to its linear partial structure that favors the formation of junction zones. Thus, it is possible to suppose that in native starch with low crystallinity, a large number of junction areas are formed in an appropriate energy state after gelatinization, showing greater crystallinity of gelatinized starch than of the native starch granules.

b)

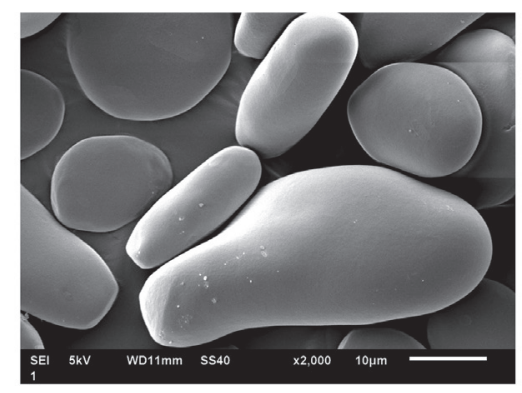

d)

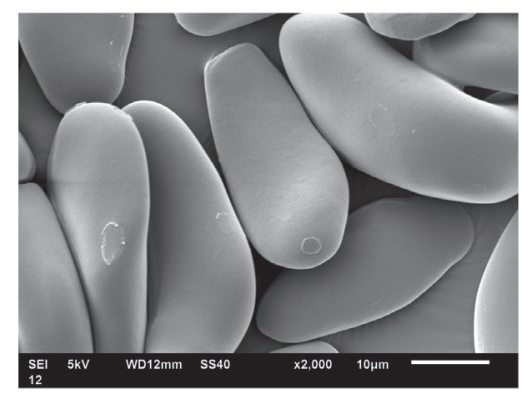

Fig. 1. Scanning electron micrographs of green banana (Musa paradisiaca L.) starch at magnification of 2000x: a) native starch, b) sample in trial 1 , c) sample in trial 8, and d) sample in trial 12 
The apparent amylose contents of heat-and-moisturetreated starch were affected by the temperature and moisture used in the treatment. The sample of trial 8 contained $58.78 \mathrm{~g} / 100 \mathrm{~g}$ apparent amylose, which was higher than the value found in the native starch $(53.70 \mathrm{~g} / 100 \mathrm{~g})$, confirming a direct relationship between temperature and moisture and apparent amylose. When the experimental data for water absorption index and swelling power were evaluated, no changes were observed when lower temperatures and moisture were used, with values similar to native starch $(1.81 \mathrm{~g} / \mathrm{g}$ and $13.66 \mathrm{~g} / \mathrm{g}$, respectively). Higher temperatures and moisture levels led to a decrease in solubility compared to native starch (9.85\%), reaching a value of $5.56 \%$.

Mathematical models in the statistical analysis were fitted to the experimental data of the apparent amylose content $\left(w_{\text {Aap }}\right)$, water absorption index (WAI), swelling power (SP) and solubility index (SI), with the aim to evaluate the behaviour of these properties as a function of the process variables. After regression analysis, the non-significant terms were eliminated. The models (Eqs. 2-5) demonstrated no significant effect of the variable time for all evaluated properties. Although $\mathrm{R}^{2}$ values can be considered low for some models, the non-significant lack of fit $\left(\mathrm{F}_{\mathrm{aj}}\right)$ demonstrated that they are suitable for describing the behaviour of these properties. Graphs were generated from the models to better understand the influence of variables in the process (Fig. 2).

$W_{\text {Aap }}=21.22+0.16 x+0.15 w_{m}$

$\left(R^{2}=0.47\right.$; non-significant lack of fit)

$\mathrm{WAI}=2.30+0.01 \mathrm{x}-0.17 w_{\mathrm{n}}+0.004 w_{\mathrm{m}}{ }^{2}$

$\left(\mathrm{R}^{2}=0.71\right.$; non-significant lack of fit)
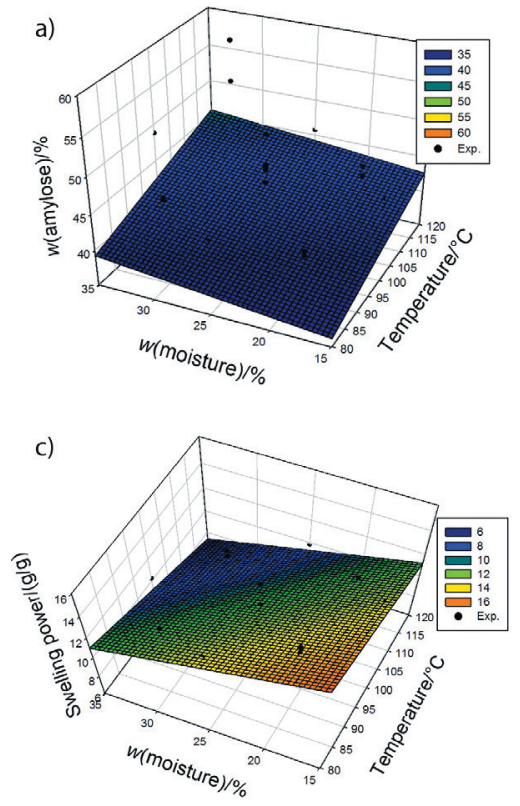

$\mathrm{SP}=24.84-0.09 x-0.19 w_{m}$

$\left(R^{2}=0.57\right.$; non-significant lack of fit)

$\mathrm{SI}=61.47-1.03 \mathrm{x}-0.06 w_{\mathrm{m}}+0.005 \mathrm{x}^{2}$

$\left(R^{2}=0.78\right.$; non-significant lack of fit)

where $\mathrm{x}$ is temperature in ${ }^{\circ} \mathrm{C}$ and $w_{\mathrm{m}}$ is moisture content.

Fig. 2a shows the variation of apparent amylose content as a function of the variables of the model. The increase in apparent amylose as a function of temperature and moisture content is probably due to the increased degradation of the outer chains of amylopectin, thus forming linear chains that were detected as amylose molecules (28). The results of water absorption index (Fig. 2b) may be due to the breakdown of the double helix structures held by hydrogen bonds during heating, thus leading to the release of hydroxyls to bind to water molecules, increasing the water absorption index (9). With the increase of linear helical structures there is an increase in $\mathrm{OH}$ exposure susceptible to the formation of more energetic and stable hydrogen-polymer/polymer bonds than those originally present in the original amylose and amylopectin structures, decreasing the solubility of the starch. The hydrogen bonds ( 2 to $7.9 \mathrm{kcal} / \mathrm{mol}$ ) are directional, meaning that in a structure that favours the formation of parallel bond, the energy and, consequently, the stability of this bond tend to be larger. In addition, the low humidity at which HMT is conducted prevents water from occupying these binding sites preferentially, as would be expected because of its high permittivity and dielectric constant. When high temperatures are used, even at low water content, the gelatinization with consequent leaching of the amylose and amylopectin occurs, favoring the solubilization of the starch. Moreover, after HMT, the granule size and agglomerates facilitate the water absorption, thus
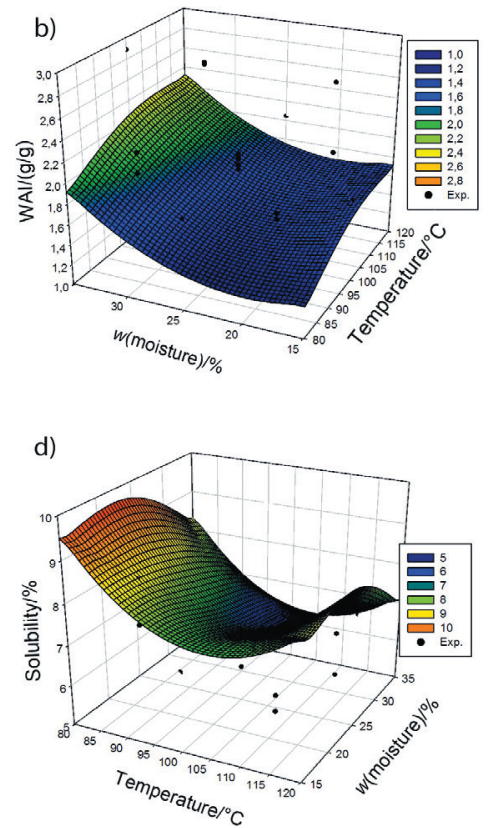

Fig. 2. Response surfaces of: a) apparent amylose content (Eq. 2), b) water absorption index (Eq. 3), c) swelling power (Eq. 4), and d) solubility index (Eq. 5). • Experimental data 
increasing water absorption index (29). An opposite trend was observed for swelling power (Fig. 2c), once the pregelatinization of starch with HMT results in reduction of the hydration capacity by the absence of granular structure. The swelling power is positively correlated with the size and distribution of the starch granules (30). This was confirmed by the micrographs of heatand-moisture-treated samples, which showed a uniform granule distribution at mild temperature and moisture conditions, in which the swelling power remained close to that observed in native starch. In solubility analysis, an opposite trend was observed, due to the retrogradation of pregelatinized amylose during HMT. The response surface graph (Fig. 2d) shows a point of minimum response, possibly due to pregelatinization occurring at higher moisture and intermediate temperature values. The swelling power and solubility index of the physically modified starch change because of the internal rearrangement of the granules, providing greater interactions between the functional groups of starch, increased crystallinity, formation of amylose-lipid complexes and structural rearrangements between amylose and amylopectin chains (4). According to BeMiller and Huber (4), these physical variations within the granules and the breaking of double helices that reduces granular stability may be responsible for the decrease in starch dilatability and solubility at higher temperatures.

\section{Pasting properties of heat-and-moisture-treated starch}

The modified starch viscosity profile is shown in Fig. 3. The sample in trial 12 showed a more pronounced drop of maximum viscosity (1348 $\mathrm{mPa} \cdot \mathrm{s}$ ) when compared to the native starch ( $9210 \mathrm{mPa} \cdot \mathrm{s})$, which was affected by the higher treatment time at intermediate temperatures and moisture levels. In all trials modified starch had higher pasting temperature than the native starch $\left(71.55^{\circ} \mathrm{C}\right)$, with a higher value observed for the sample in trial $8\left(84.35^{\circ} \mathrm{C}\right)$. For final viscosity and setback viscosity, higher values were observed in the samples subjected to lower moisture and temperature conditions.

Statistical adjustments were made from the experimental data with the purpose of generating mathematical models capable of predicting the behaviour of the response variables maximum viscosity $\left(\eta_{\max }\right)$, pasting temperature $\left(x_{p}\right)$, final vis$\operatorname{cosity}\left(\eta_{\text {final) }}\right)$, and setback viscosity $\left(\eta_{\text {setback }}\right)$ of the hydrothermally modified starch (Eqs. 6-9).

$$
\begin{aligned}
& \eta_{\max }=65338-792.8 x-505.96 y-1097.37 w_{m}+ \\
& +3.5 x^{2}+14.38 y^{2}+19.83 w_{m}{ }^{2}
\end{aligned}
$$

$\left(R^{2}=0.83\right.$; non-significant lack of fit)

$$
\mathrm{x}_{\mathrm{p}}=53.92+0.16 \mathrm{x}+0.20 \mathrm{y}+0.31 w_{\mathrm{m}}
$$

$\left(R^{2}=0.73\right.$; non-significant lack of fit)

$$
\eta_{\text {final }}=22810-173.82 \mathrm{x}
$$

$\left(R^{2}=0.34\right.$; non-significant lack of fit)

$$
\eta_{\text {setback }}=15899-99.13 x-141.02 w_{\mathrm{m}}
$$

$\left(R^{2}=0.49\right.$; non-significant lack of fit) where $\mathrm{x}$ is temperature in ${ }^{\circ} \mathrm{C}$, $\mathrm{y}$ is time in $\mathrm{h}$, and $w_{\mathrm{m}}$ is moisture content in \%.

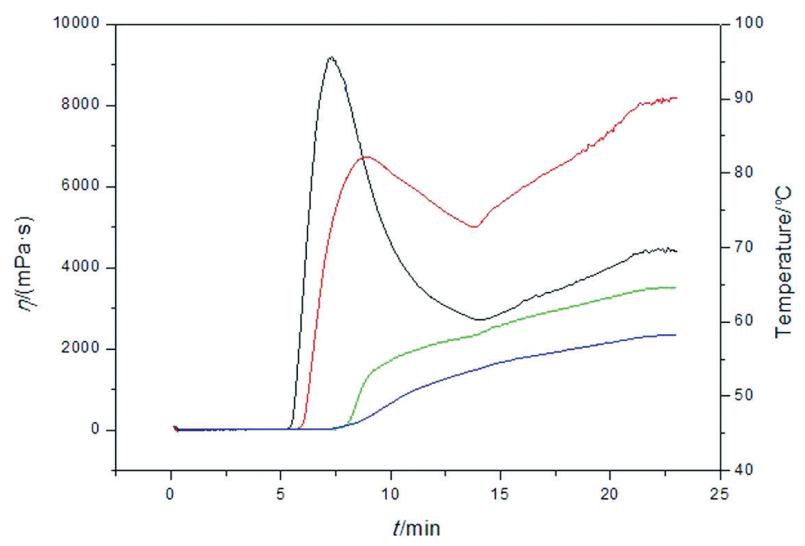

Fig. 3. Effect of modification on green banana starch pasting profile

Figs. $4 a, 4 b$ and $4 c$ show the minimum point for the values of maximum viscosity, which was reached when using intermediate values of HMT variables. This was a result of the pregelatinization of starch subjected to hydrothermal treatment, where a small fraction of the starch components, mainly amylose molecules, have already leached and undergone retrogradation during cooling, thus increasing the resistance to gelatinization. The pasta viscosity is related to the level of degradation of starch granule after the hydrothermal treatment, which then becomes less swollen, not being able to reach the same viscosity as the native granules, resulting in a decrease of viscosity (31). With the increase in inter- and intramolecular interactions, higher temperatures are required for breaking, increasing the pasting temperature (22), which can be observed in Figs. 4d, 4e and 4f. Under these conditions, starch had higher crystallinity than native starch, requiring more energy to dissociate the intramolecular hydrogen bonds, resulting in higher pasting temperature. The results of final viscosity (Fig. $4 \mathrm{~g}$ ) are probably due to leaching of the granule and exposure of amylose and amylopectin molecules to high temperatures. For setback viscosity (Fig. 4h), higher values were observed at lower temperatures and moisture levels. This increase in gel formation by the association of starch molecules after modification is due to the high apparent amylose content of starch. Amylose is, besides representing the largest starch fraction of this study, also the main molecule involved in the first stage of retrogradation, which was analyzed by rapid visco analyzer.

\section{Thermal analysis of heat-and-moisture-treated starch}

A gradual increase in the initial and pasting temperatures was observed in the modified starch, when compared to the native starch at the gelatinization level, with higher values in the sample in trial $8\left(83.59\right.$ and $87.92^{\circ} \mathrm{C}$, respectively) than in the native starch $\left(69.22\right.$ and $74.10^{\circ} \mathrm{C}$, respectively). The final temperature required for gelatinization was affected by the starch characteristics since no change was observed (84.83 
a)
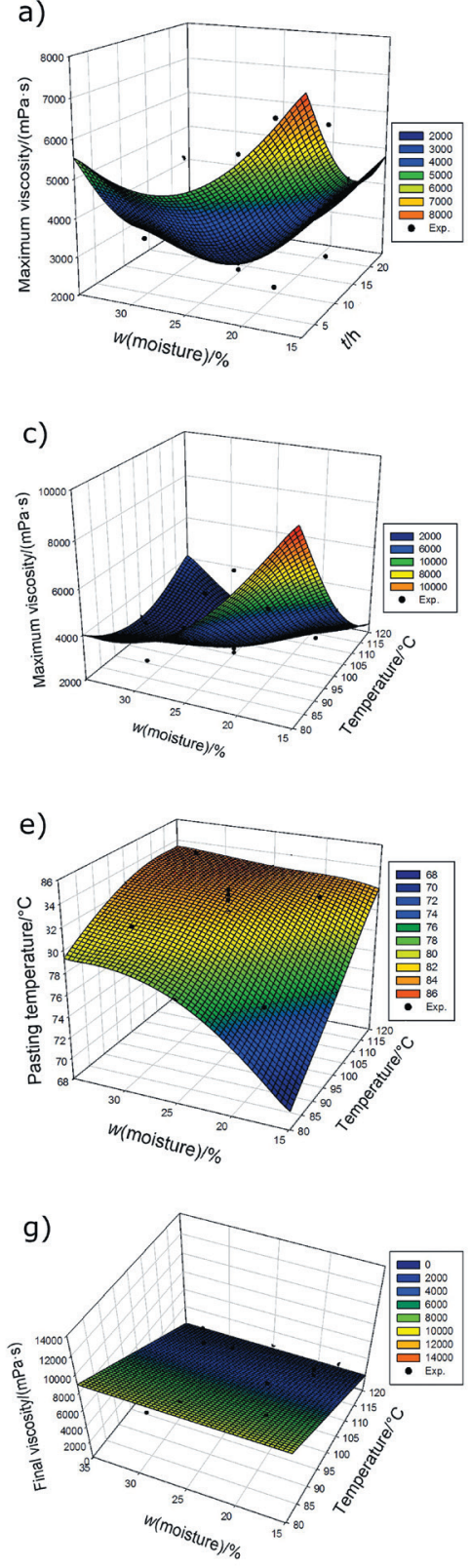

b)

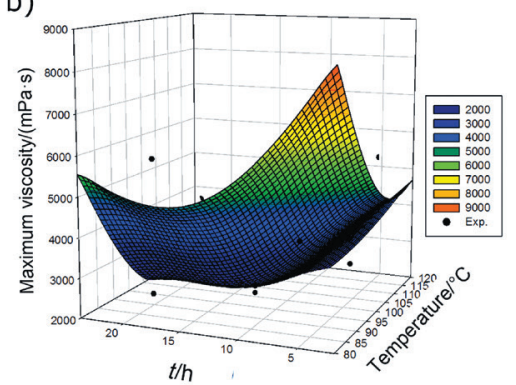

d)
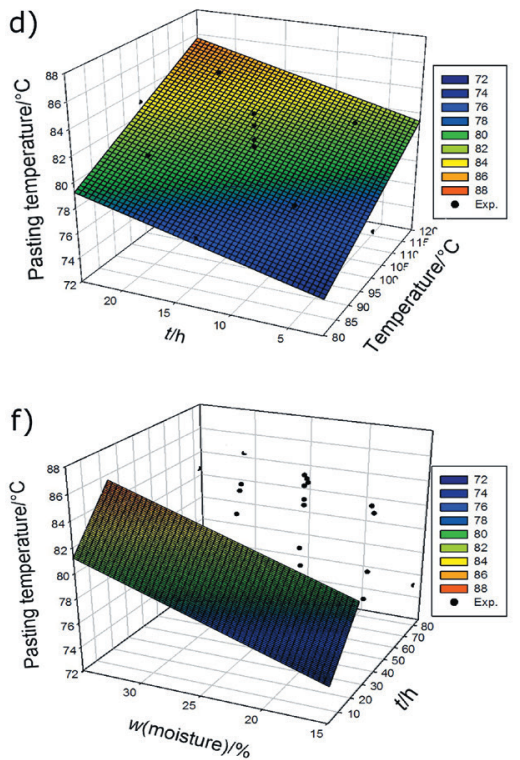

h)

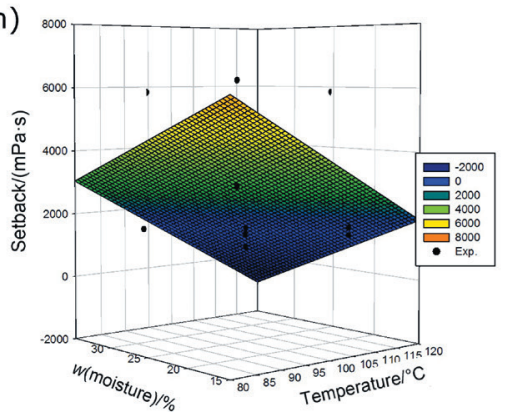

Fig. 4. Response surfaces of the pasting properties measured with rapid visco analyzer: a-c) maximum viscosity (Eq. 6), d-f) pasting temperature (Eq. 7), g) final viscosity (Eq. 8), and h) retrograde of starch (Eq. 9). Experimental data

${ }^{\circ} \mathrm{C}$ ) under mild moisture and temperature conditions (sample in trial 1) compared to the native starch $\left(85.21^{\circ} \mathrm{C}\right)$. Opposite trend was observed for gelatinization enthalpy, with values inversely proportional to the temperature of the modification process. Melting curves, with endothermic peaks, were observed for all samples, which are associated with the gelatinization process.

When analyzing initial temperature (Eq. 10), pasting temperature (Eq. 11) and final temperature (Eq. 12) in the models, linear behaviour was observed as a function of temperature and moisture content, with moisture having the most significant effect. For the gelatinization enthalpy (Eq. 13), a quadratic term was observed for the variable moisture, and a linear term for temperature, which was the most significant.

$$
X_{i}=46.72+0.15 x+0.55 w_{m}
$$

$\left(R^{2}=0.65\right.$; non-significant lack of fit)

$X_{p}=46.68+0.15 x+0.21 y+0.53 w_{m}$

$\left(R^{2}=0.80\right.$; non-significant lack of fit)

$X_{f}=64.10+0.15 x+0.13 y+0.37 w_{m}$

$\left(R^{2}=0.86\right.$; non-significant lack of fit)

$\Delta H=9.06-0.17 \mathrm{x}+1.75 w_{\mathrm{m}}-0.04 w_{\mathrm{m}}^{2}$ 
where $X_{i}, X_{p}$ and $X_{f}$ are initial, pasting and final temperature in ${ }^{\circ} \mathrm{C}$, respectively, $\mathrm{y}$ is time in $\mathrm{h}, \Delta H$ is gelatinization enthalpy and $w_{\mathrm{m}}$ is moisture content in \%.

The increase in the initial temperature of gelatinization with an increase in temperature and moisture content (Fig. 5a) shows that the physical modification altered the structure of native starch, leading to the pregelatinization of the granules, requiring a greater amount of energy to start the gelatinization process. This behaviour may also explain the changes in pasting temperatures (Figs. $5 b, 5 c$ and $5 d$ ). The results of final temperature (Figs. $5 e, 5 f$ and $5 g$ ) are due to the new arrangements in the structure of the starch molecules, because of the presence of retrograded amylose, leading to greater cohesiveness in the paste formed and greater strength of the hydrogen bonding between amylose and amylopectin and water molecules, making it difficult to dissociate, thus increasing the final temperature (9).

The graph of enthalpy of gelatinization (Fig. $5 \mathrm{~h}$ ) shows the point of maximum response. This variable reflects the amount of energy required to break the intramolecular bonds of the starch granules during gelatinization. Since thermal analysis was done between 40 and $120^{\circ} \mathrm{C}$, the measured enthalpy is only associated with gelatinization process of the native
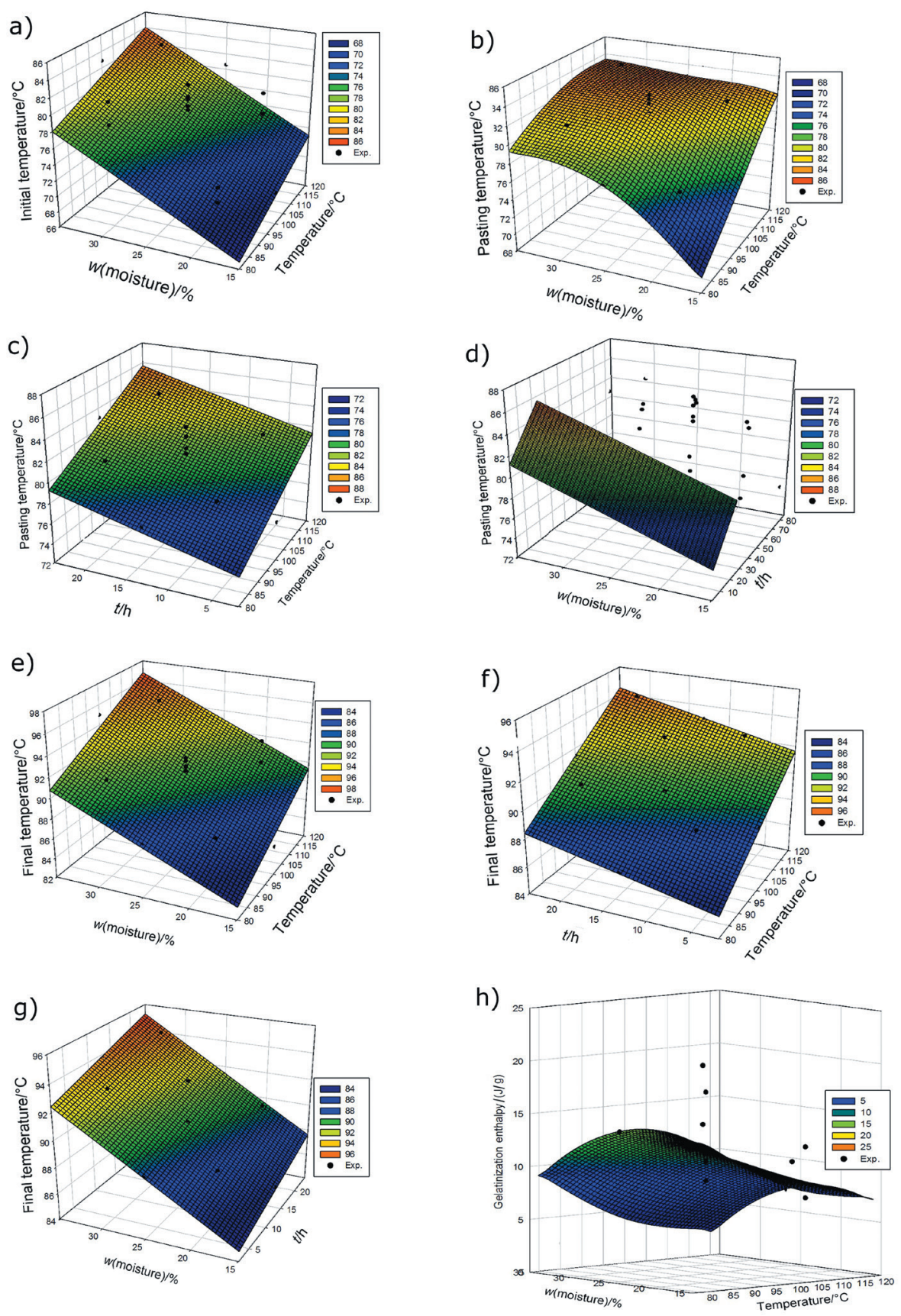

Fig. 5. Response surfaces of the thermal analysis regarding the parameters: a-c) initial temperature (Eq. 10), d-f) pasting temperature, (Eq. 11), g) final temperature (Eq. 12) and h) gelatinization enthalpy $\Delta H$ (Eq. 13). Experimental data 
starch granules that resisted the hydrothermal treatment. In view of that, much higher temperatures would be required to verify the energy involved in the melting of the junction zones resulting from the crystallization in the pregelatinized starch, given the high concentration of apparent amylose present in the studied starch.Thus, the more severe the HMT, the lower the amount of remaining native granules, resulting in lower gelatinization enthalpy.

\section{Digestibility in vitro of heat-and-moisture-treated starch}

The digestibility in vitro of resistant and non-resistant content heat-and-moisture-treated green banana starch was measured. The samples subjected to the lowest temperature and moisture levels gave the highest resistant starch content, as observed in the sample in trial 1 with $51.63 \mathrm{~g} / 100 \mathrm{~g}$, which was higher than the native starch $(37.71 \mathrm{~g} / 100 \mathrm{~g})$. However, a marked drop was observed in the sample in trial 8 with an increase in time and temperature, which had the lowest resistant starch content $(0.96 \mathrm{~g} / 100 \mathrm{~g})$. Great changes were observed in non-resistant starch contents, with the lowest (11.42 $\mathrm{g} / 100 \mathrm{~g}$ ) and highest $(97.23 \mathrm{~g} / 100 \mathrm{~g})$ values for the samples in trials 1 and 8, respectively.

A linear model as a function of temperature $(x)$ and moisture $\left(w_{\mathrm{m}}\right)$ was obtained for the non-resistant starch content $\left(w_{\text {nrs }}\right)$ (Eq. 14), and despite the low $R^{2}$ value, the significance of the model associated with lack of adjustment $\left(F_{a j}\right)$ shows that the model is adequate and satisfactorily describes the behavior trend for non-resistant starch content.

$$
w_{\text {nrs }}=-138.01+1.64 x+1.90 w_{m}
$$

$\left(R^{2}=0.67\right.$; non-significant lack of fit)

The content of non-resistant starch (Fig. 6) can be due to the effect of pregelatinization of starch during the hydrothermal treatment, again showing that the green banana starch granule is relatively fragile under rigorous hydrothermal conditions. The hydrothermal treatment leads to a disorganization in the semicrystalline structure of the starch granule,

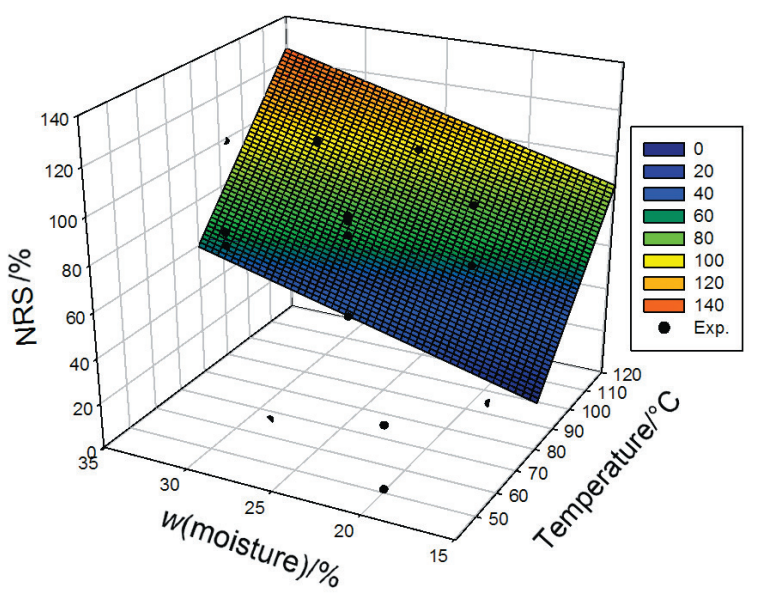

Fig. 6. Response surface of non-resistant starch (NRS) content modified by heat treatment with low humidity according to the adjusted model (Eq. 14). • Experimental data indicating that type B polymorphism is critical for the digestion property, which was observed in the present study, since a small change in the crystalline region of the modified starch was detected by X-ray diffraction (1). Thus, the hydrothermal treatment favours the reduction of non-resistant starch fractions when subjected to low temperature and moisture levels, which is desirable, whereas degradation of the granular structure can occur at high temperature and moisture levels, greatly increasing the amount of this starch fraction, thus modifying the properties of digestibility inherent to banana starch.

\section{CONCLUSIONS}

Mathematical models describing the behaviour of modified starch properties as a function of the evaluated variables were obtained through statistical analysis. The variables time and temperature had a significant effect on the physicochemical, rheological and digestibility properties of starch. The heat-moisture treatment (HMT) has generated modified starch with very distinct properties ranging from the high tendency to retrograde and high viscosity to the low tendency to retrograde and low viscosity. Reduction of resistant starch content was great in the samples subjected to higher temperature and moisture levels, but it was higher in milder treatments, evidencing that the green banana starch granule is relatively fragile under the most rigorous hydrothermal conditions. The results showed that heat-moisture treatment can be an effective method to improve heat stability and shear stress resistance of banana starch.

\section{ACKNOWLEDGEMENTS}

The authors are grateful to Coordination for the Improvement of Higher Education Personnel (CAPES) for the financial support and scholarship.

\section{REFERENCES}

1. Agama-Acevedo E, Nuñez-Santiago MC, Alvarez-Ramirez J, Bello-Pérez LA. Physicochemical, digestibility and structural characteristics of starch isolated from banana cultivars. Carbohydr Polym. 2015;124:17-24.

https://doi.org/10.1016/j.carbpol.2015.02.003

2. Zhang P, Whistler RL, BeMiller JN, Amaker BR. Banana starch: Production, physicochemical properties, and digestibility A review. Carbohydr Polym.2005;59(4):443-58.

https://doi.org/10.1016/j.carbpol.2004.10.014

3. Gao F, Li D, Bi C, Mao Z, Adhikari B. Preparation and characterization of starch crosslinked with sodium trimetaphosphate and hydrolyzed by enzymes. Carbohydr Polym. 2014;103:310-8.

https://doi.org/10.1016/j.carbpol.2013.12.028

4. BeMiller JN, Huber KC. Physical modification of food starch functionalities. Annu Rev Food Sci Technol. 2015;6:19-69. https://doi.org/10.1146/annurev-food-022814-015552 
5. Varatharajan V, Hoover R, Liu Q, Seetharaman K. The impact of heat-moisture treatment on the molecular structure and physicochemical properties of normal and waxy potato starches. Carbohydr Polym. 2010;81(2):466-75.

https://doi.org/10.1016/j.carbpol.2010.03.002

6. Hormdok R, Noomhorm A. Hydrothermal treatments of rice starch for improvement of rice noodle quality. LWT - Food Sci Tech. 2007;40(10):1723-31.

https://doi.org/10.1016/j.lwt.2006.12.017

7. Yadav BS, Guleria P, Yadav RB. Hydrothermal modification of Indian water chestnut starch: Influence of heat-moisture treatment and annealing on the physicochemical, gelatinization and pasting characteristics. LWT - Food Sci Technol. 2013;53(1):211-7.

https://doi.org/10.1016/j.lwt.2013.02.007

8. Hoyos-Leyva JD, Bello-Pérez LA, Agama-Acevedo E, AlvarezRamirez J. Optimising the heat moisture treatment of Morado banana starch by response surface analysis. Starch/ Starke. 2015;67(11-12):1026-34.

https://doi.org/10.1002/star.201500149

9. Singh M, Adedeji AA. Characterization of hydrothermal and acid modified proso millet starch. LWT - Food Sci Technol. 2017;79:21-6.

https://doi.org/10.1016/j.lwt.2017.01.008

10. Moorthy SN. Extraction of starches from tuber crops using ammonia. Carbohydr Polym. 1991;16(4):391-8.

https://doi.org/10.1016/0144-8617(91)90057-J

11. Chung HJ, Liu Q, Hoover R. Impact of annealing and heatmoisture treatment on rapidly digestible, slowly digestible and resistant starch levels in native and gelatinized corn, pea and lentil starches. Carbohydr Polym.2009;75(3):436-47. https://doi.org/10.1016/j.carbpol.2008.08.006

12. AOAC Official Method 945.15. Moisture in cereal adjuncts. Rockville, MD, USA: AOAC International; 1997.

13. AOAC Official Method 923.03. Ash of flour. Rockville, MD, USA: AOAC International; 1997.

14. AOAC Official Method 945.18. Cereal adjuncts. Rockville, MD, USA: AOAC International; 1997.

15. AOAC Official Method 945.27. pH of brewing suagars and syrups. Rockville, MD, USA: AOAC International; 1997.

16. Bligh EG, Dyer WJ. A raipd method of total lipid extraction and purification. Can J Physiol Pharm. 1959;37(8):911-7.

17. Martinez C, Cuevas F, Medina LM. Evaluation of the culinary and rice milling quality. Cali, Colombia: Centro Internacional de Agricultura Tropical (CIAT), Series 04SR-07.01; 1989 (in Spanish).

18. Kaitaranta JK, Lamppu R, Linko RR. Amino acid content of Baltic herring and rainbow trout roe. J Agric Food Chem. 1980;28(5):908-11.

https://doi.org/10.1021/jf60231a028
19. Schoch TJ. Swelling power and solubility of granular starches. In: Whistler RL, Wolfrom ML, editors. Methods in carbohydrate chemistry, Vol. 4. New York, NY, USA: Academic Press; 1964. pp. 106-8.

20. Hart Weber F, Collares-Queiroz FP, Chang YK. Physicochemical, rheological, morphological, and thermal characterization of normal, waxy and high amylose corn starches. Ciênc Tecnol Aliment. 2009;29(4):748-53 (in Portuguese). https://doi.org/10.1590/S0101-20612009000400008

21. Scanning Electron Microscope SEM Control User Interface, v. 2.24, Hitachi High Technologies, Tokyo, Japan; 2014.

22. SAS University Edition, SAS Institute Inc. Cary, NC, USA; 2017. Available from: https://www.sas.com.

23. de Souza Rocha T, de Almeida Carneiro AP, Landi Franco CM. Effect of enzymatic hydrolysis on some physicochemical properties of root and tuber granular starches. Cienc Tecnol Aliment. 2010;30(2):544-51.

https://doi.org/10.1590/S0101-20612010000200039

24. Knutson CA. Evaluation of variations in amylose-iodine absorbance spectra. Carbohydr Polym. 1999;42(1):65-72.

25. Pepe LS, Moraes J, Albano KM, Telis VRN, Franco CML. Effect of heat-moisture treatment on the structural, physicochemical, and rheological characteristics of arrowroot starch. Food Sci Technol Int. 2016;22(3):256-65. https://doi.org/10.1177/1082013215595147

26. Liu J, Wang B, Lin L, Zhang J, Liu W, Xie J, Ding Y. Functional, physicochemical properties and structure of cross-linked oxidized maize starch. Food Hydrocoll. 2014;36:45-52. https://doi.org/10.1016/j.foodhyd.2013.08.013

27. Sukhija S, Singh S, Riar CS. Physicochemical, crystalline, morphological, pasting and thermal properties of modified lotus rhizome (Nelumbo nucifera) starch. Food Hydrocoll. 2016;6:50-8.

https://doi.org/10.1016/j.foodhyd.2016.03.013

28. Vaz Gonçalves MF, Silveira Sarmento SB, dos Santos Dias $\mathrm{CT}$, Marquezini N. Heat moisture treatment of sweet potato starch (Ipomoea batatas L.) in microwave oven. Cienc Tecnol Aliment. 2009;29(2):270-6.

https://doi.org/10.1590/S0101-20612009000200005

29. Naidoo K, Amonsou EO, Oyeyinka SA. In vitro digestibility and some physicochemical properties of starch from wild and cultivated amadumbe corms. Carbohydr Polym. 2015; 125:9-15.

https://doi.org/10.1016/j.carbpol.2015.02.066

30. de la Torre Gutiérrez L, Chel-Guerrero LA, Betancur-Ancona D. Functional properties of square banana (Musa balbisiana) starch. Food Chem. 2008;106(3):1138-44. https://doi.org/10.1016/j.foodchem.2007.07.044

31. Teba CS, Ascheri JLR, Carvalho CWP. Effect of the extrusion parameters on the pasting properties of precooked rice and bean pasta. Alim Nutr. 2009;20(3):411-26 (in Portuguese). 